\title{
Cumplimiento de los programas de desarrollo social y ambiental de la empresa Minera Barrick en la Unidad de Producción Pierina -Huaraz
}

\section{Compliance with the social and environmental development programs of the Minera Barrick company in the Pierina-Huaraz Production Unit}

\author{
Luis Alfredo Orihuela Salazar ${ }^{1}$, Carlos Cabrera Carranza², Sixto Vidal Aramburu Rojas ${ }^{3}$
}

Recibido: 18/11/2019 - Aprobado: 01/10/2021 - Publicado: 23/12/2021

\begin{abstract}
RESUMEN
La investigación realizada planteó analizar el impacto sobre la realización de los programas de desarrollo social y ambiental de la empresa Minera Barrick para lo cual se enfocó la Unidad de Producción Pierina -Huaraz. Para ello se contó con una investigación de tipo elemental, haciendo uso de un diseñamiento investigativo de naturaleza cuantitativa siguiendo en estricto cumplimiento el rigor académico. El universo considerado en el presente estudio fue la comunidad de Cuncashca, Mareniyoc, Mataquita y Atupa, correspondientes a Jangas, pertenecientes a Huaraz las cuales tienen una población de 767 habitantes, distribuidas en 330 viviendas, para lo que en referencia al estudio, se consideró una muestra de 75 familias de las localidades cercanas, aplicando un instrumento de 15 preguntas dicotómicas, que tomo en consideración los aportes significativos que se brinda a las comunas aledañas, lo correspondiente a los indicadores de sostenibilidad, así como también la relación positiva y simbiótica con la comuna aledaña. Se tiene que los hallazgos más destacables, para la comunidad Cuncashca, un $75 \%$ señala que a través del Programa PIRSA implementado por la empresa minera se dio pasos firmes hacia la sostenibilidad de desarrollo, que emprende la empresa Barrick, de igual forma un $72.5 \%$ de los encuestados de la comunidad Mareniyoc señalan lo mimo a igual que $65.5 \%$ de Atupa y el $63.5 \%$ respectivamente Mataquita. Es importante también mencionar en aras de la verdad y la transparencia necesaria en todo proceso de investigación científica que un porcentaje reducido de pobladores de las zonas aledañas al asentamiento minero, expresaron que el apoyo por parte de las autoridades de la minera, no fue el adecuado en cada comunidad cercana; los resultados obtenidos evidenciaron que hay un sector de dicha población que no reconoce el aporte de la minera, sin embargo, el gran porcentaje expresó que si lo hay. Por lo que se concluye que el alcance de los programas de la empresa minera, se traducen en proyectos de inversión sostenibles, y un incentivo que va más allá del aspecto netamente economista, pues está enfocado seriamente en la búsqueda de brindar mejores condiciones de vida a la población en tanto se sientan las bases para un desarrollo sostenible para todas las comunas aledañas al asentamiento minero enfocado en el marco del estudio.
\end{abstract}

Palabras claves: Desarrollo sostenible; programas sociales; Mina Pierina; empresa minera Barrick.

\section{ABSTRACT}

The research carried out proposed analyzing the impact on the implementation of the social and environmental development programs of the Minera Barrick company, for which the Pierina-Huaraz Production Unit focused. For this, an elementary type of investigation was used, making use of an investigative design of a quantitative nature, in strict compliance with academic rigor. The universe considered in the present study was the community of Cuncashca, Mareniyoc, Mataquita and Atupa, corresponding to Jangas, belonging to Huaraz which have a population of 767 inhabitants, distributed in 330 homes, for which in reference to the study, it was considered a sample of 75 families from nearby towns, applying an instrument of 15 dichotomous questions, which I take into consideration are those related to the significant contributions that are provided to neighboring communities, corresponding to sustainability indicators, as well as the relationship positive and symbiotic with the neighboring commune. The most remarkable findings are to be found, for the Cuncashca community $75 \%$ indicate that through the PIRSA Program implemented by the mining company firm steps were taken towards the sustainability of development, which is undertaken by the Barrick company, in the same way a $72.5 \%$ of Mareniyoc community respondents point out the same as $65.5 \%$ from Atupa and $63.5 \%$ respectively from Mataquita. It is also important to mention for the sake

1 Universidad Nacional Mayor de San Marcos, Facultad de Ingeniería Geológica, Minera, Metalúrgica y Geográfica, Lima, Perú. Ingeniero Metalúrgico. Docente del Departamento de Ingeniería Metalúrgica.

Autor para correspondencia: lorihuelas@unmsm.edu.pe - ORCID: https://orcid.org/0000-0003-3114-8318

2 Universidad Nacional Mayor de San Marcos, Facultad de Ingeniería Geológica, Minera, Metalúrgica y Geográfica, Lima, Perú. Docente del Departamento de Ingeniería Geográfica. E-mail: ccabrerac@unmsm.edu.pe - ORCID: https://orcid.org/0000-0002-5821-5886

3 Universidad Nacional Mayor de San Marcos, Facultad de Ingeniería Geológica, Minera, Metalúrgica y Geográfica, Lima, Perú. Docente del Departamento de Ingeniería Metalúrgica.

E-mail: varamburur@unmsm.edu.pe - ORCID: https://orcid.org/0000-0001-5958-6382 
of truth and the necessary transparency in any scientific research process that a small percentage of residents of the areas surrounding the mining settlement expressed that the support from the mining authorities was not adequate. In each nearby community, the results obtained showed that there is a sector of said population that does not recognize the contribution of the mining company, however, the large percentage expressed that there is. Therefore, it is concluded that the scope of the mining company's programs translates into sustainable investment projects, and an incentive that goes beyond the purely economic aspect, since it is seriously focused on the search to provide better living conditions to the population while laying the foundations for sustainable development for all the communes surrounding the mining settlement focused in the framework of the study.

Keywords: Sustainable development; social programs; Pierina Mine; Barrick mining company.

\section{INTRODUCCIÓN}

Cuando se hace referencia al bienestar social de los habitantes de un pais, se toma en consideración un conjunto amplio de variables, como políticas de estados, salud, economía entre otros, donde de alguna forma, todos los sectores económicos y sociales tienen una participación importante. Tomando en cuenta ello, se tiene que, en gran media, el desarrollo social conlleva o tiene como propósito el consolidar la igualdad de condiciones para el acceso al bienestar, siendo esto más notables en las sociedades.

De manera particular, en el Perú uno de los sectores determinantes para el desarrollo social, es el industrial y empresarial, teniendo una participación de gran importancia, las empresas mineras. Es por esta razón, que, para fines de este artículo, se ha querido realizar un análisis sobre el impacto de los programas de desarrollo social y ambiental que estas empresas desarrollan en el país, y como ello se traduce en resultados para los avances en el Perú, mas específicamente a la empresa minera Barrick en la Unidad de Producción Pierina -Huaraz, teniendo como objetivo el de analizar el impacto sobre el cumplimiento de los programas de desarrollo social y ambiental de la empresa Minera Barrick en la Unidad de Producción Pierina -Huaraz. Cabe señalar que la inspiración de esta investigación se basa en un trabajo de tesis realizado por (Luis Alberto Orihuela Salazar, 2019) que tiene por título "la responsabilidad social y ambiental de la industria minera en el Perú. Caso: Minera Barrick Misquichilca-Pierina”, no obstante, el resultado de este estudio en particular representa un desglose y análisis producto de lo experimentado en la citada investigación, por lo que será nombrada y traída a acotación para reforzar diversos puntos.

\section{METODOS}

Este artículo de investigación se caracteriza por estar enmarcado en un estudio de tipo básico con un diseño no experimental, de naturaleza cuantitativa, y de nivel descriptivo, cuya finalidad es la de realizar una pesquisa investigativa que brinde datos sobre las variables en estudio, para así poder analizar y emitir una conclusión respectiva.

\subsection{Población de estudio}

Para (Hernández-Sampieri, 2014) la población es el "conjunto de todos los casos que concuerdan con determinadas especificaciones" (p.174), para fines de esta investigación, se contó como población a las comunidades circundantes a la mina, que las cuales son la comunidad de Cuncashca, Mareniyoc, Mataquita y Atupa, comunidades del distrito de Jangas, Provincia de Huaraz las cuales tienen una población de 767 habitantes, distribuidas en 330 viviendas.

\subsection{Muestra}

Según (Sergio Carrasco Díaz, 2005) "la muestra de una investigación cuantitativa se refiere a un corte sociodemográfico de la población para crear un grupo específico que se corresponda con el público objetivo y así poder comprender su comportamiento para aplicarlo en una campaña" (p.34). Para fines de estudio, la muestra escogida fue calculada en base a la fórmula desarrollada por (Spiegel \& Stephens, 2009) es la siguiente:

$$
n=\frac{N \times Z_{a}^{2} \times p \times q}{d^{2} \times(N-1)+Z_{a}^{2} \times p \times q}
$$

Consecuentemente, se calculó la muestra con base a información suministrada por el (INEI, 2019) la cual se señala en la siguiente tabla 1.

Tabla 1. Números de viviendas a entrevistar

\begin{tabular}{lccc}
\hline Comunidad & $\begin{array}{c}\text { Población residente } \\
\text { (Hab.) }\end{array}$ & $\begin{array}{c}\text { Número de } \\
\text { viviendas }\end{array}$ & $\begin{array}{c}\text { No. viviendas } \\
\text { para entrevistar }\end{array}$ \\
\hline Cuncashca & 57 & 37 & 8 \\
Mareniyoc & 143 & 69 & 16 \\
Mataquita & 481 & 177 & 40 \\
Atupa & 86 & 47 & 11 \\
Total & 767 & 330 & 75 \\
\hline
\end{tabular}

Fuente: (INEI, 2019)

Por lo que luego de hacer el cálculo se determina que el total de viviendas a entrevistar son 75 viviendas distribuidas en las 4 comunidades.

\subsection{Técnicas de recolección de datos}

Para el autor, (Juan Antonio Gil Pascual, 2016) se entiende por técnicas de recogida de información al total de los medios técnicos que se emplean para recoger los datos y registrar las observaciones o facilitar el tratamiento. A continuación, se presenta la tabla 2 con información relevante al respecto:

Tabla 2. Números de viviendas a entrevistar

\begin{tabular}{ll}
\hline Técnicas & Instrumentos \\
\hline Análisis de documental & Ficha bibliográfica \\
Encuestas & Cuestionario dicotómico de 15 preguntas \\
\hline
\end{tabular}

Fuente: Elaboración propia 


\subsection{Análisis de confiabilidad y validez}

A través de la aplicación piloto de una prueba se establecerá la confiabilidad del cuestionario diseñado, para luego aplicar la prueba de Kuder-Richardson, debido a que corresponde para el diseño del mismo.

$$
r_{20}=\left(\frac{K}{K-1}\right)\left(\frac{\sigma^{2}-\sum p q}{\sigma^{2}}\right)
$$

Donde:

$\mathrm{K}=$ Número de Ítems del instrumento

$\mathrm{p}=$ Porcentaje.de personas que responde correctamente cada item

$\mathrm{q}=$ Porcentaje.de personas que responde incorrectamente cada item

$\sigma^{2}=$ Varianza total del instrumento

Ahora bien, esta prueba piloto se aplicó a 10 personas pertenecientes a la población, mas no pertenecientes a la muestra, obteniéndose así un valor de 0.76 , entrando en el rango de 0.76 a 0.89 , considerado como fuerte, o que permite afirmar que los resultados que arrojara el instrumento a utilizar serán fuertemente confiables, y permitirá una correcta medición de las variables de estudio.

\section{RESULTADOS Y DISCUSIÓN}

Posterior a la elaboración y ejecución de la técnica utilizada para la recaudación de la información pertinente, esta se analizó y se interpretó la información acumulada, tanto de tipo bibliográfica como de campo, para que luego la información recolectada se tabulara de manera organizada según dicta el adecuado procesamiento exigido por el rigor académico y científico. Este articulo por su parte, centro su interés en la empresa Minera Barrick Misquichilca Pierina, la cual tiene en desarrollo su Programa Integral de Responsabilidad Social y Ambiental (PIRSA) que no es sino un proyecto que aborda cuestiones de corte social y de ambiental generando conciencia y desarrollando acciones que coadyuven al logro paulatino de los objetivos de desarrollo sostenible planteados por los organismos internacionales (Espinoza Guadalupe, 2004).

Para sus fines planteados, esta investigación, se tomará en consideración, los datos recolectados por (Luis Alberto Orihuela Salazar, 2019) en su investigación, cuyos datos fueron procesados mediante la estadística descriptiva, haciendo uso del paquete estadístico SPSS versión 24. Es de hacer notar, los resultados aquí observados, han sido analizados tomando en consideración como marco de sustento, el enfoque de desarrollo humano, con la finalidad de abordar de forma integral, las temáticas relacionadas a las trasformaciones socioeconómicas experimentadas por el universo poblacional considerado en el marco del esfuerzo investigativo realizado.

Basado en este enfoque, se toman en consideración la realidad de la gente que habita a los alrededores del asentamiento minero y su evolución en el tiempo a causa de la acción de la empresa minera. Desde una visión investigativa y metodológica, la investigación realizada por (Luis Alberto Orihuela Salazar, 2019) aporta datos interesantes para el análisis de la investigación, pues se basa en censos poblacionales que fueron aplicados antes de la mina Pierina y luego de ella, lo que permite determinar con mayor claridad el impacto en las comunidades circundantes, obteniéndose así, los siguientes resultados:

\subsection{Características generales de las comunidades}

Es de hacer notar que las comunidades escogidas son aquellas que tienen una mayor cercanía a las operaciones en la veta aurífera que se desempeñan en la zona, las cuales son la comunidad de Cuncashca, Mareniyoc, Mataquita y Atupa, comunidades del distrito de Jangas, Provincia de Huaraz y además han logado establecer vínculos con la empresa. Se puede señalar como dato relevante que la población mayor de edad hasta las seis décadas representa la más sobresaliente en las localidades, siendo distribuida desde el 48.8\% (Atupa) a 55.9\% (Mataquita), lo que representa un promedio de $52.0 \%$ para los cuatros poblados considerados para el estudio, tomando en cuenta que, de toda la población, el $62.7 \%$ del total pertenece a la comunidad Mataquita. En referencia a la alfabetización, se tiene que de toda la población estudiada solo el $66 \%$ manifestó saber leer y escribir, teniendo un $47 \%$ en promedio de quienes tienen un nivel de estudios en primaria.

Con respecto a las características de las viviendas, se señala que el $92 \%$ de las viviendas de la región, son denominadas como propias, construidas en un $82 \%$ con material de adobe, con techos de calamina para el $83 \%$ de las viviendas, y con servicio de energía eléctrica para el $92 \%$ de las mismas. Aunque los servicios higiénicos son por la red pública $(65 \%)$, pozos sépticos $(25 \%)$ y otros $(10 \%)$ y para la cocina, en su mayoría $(81 \%)$ es leña.

En lo relativo al área económica, se puede apreciar que grandes porcentajes de las comunas aledañas al asentamiento minero se desenvuelven en actividades agrícolas, siendo en un rango comprendido entre $65.2 \%$ a $86.4 \%$, seguido por la actividad de prestación de servicios comprendido en un rango del $4.5 \%$ a $13.5 \%$; y en un porcentaje mucho menor se tiene al sector minero, con $3.4 \%$ a $10.9 \%$.

Cabe resaltar que, como hecho relevante, las comunidades estudiadas, luego de que se emprendiera las operaciones mineras de Pierina han reflejado un crecimiento demográfico superior al promedio de crecimiento en el Perú, siendo este un impacto sustancial debido a que, según datas anteriores, la población tiende a migrar de esas zonas, y justamente se expresa que la población que habita en dichas áreas territoriales migra debido a las condiciones de vida que tienen, difícil acceso a una educación de calidad, prácticamente nula atención médica, alta dificultad para acceder a la tecnología, y un aspecto no menos importante: la inexistente posibilidad de acceso a oportunidades de crecimiento personal y profesional, por lo tanto, la imposibilidad de aspirar a que sus generaciones futuras logren abrirse un camino propio sin perjudicar al resto.

Situaciones difíciles que pueden darse, aun cuando vivimos en una "civilización actualizada" en ciudades metrópoli que concentran prácticamente todo en el país, 
instituciones que supuestamente son descentralizadas, pero de ellas poco o nada funciona en provincia, y a la que muy pocos peruanos de las zonas mineras o de las zonas más alejadas del país tienen acceso. Aquí puede aplicarse la siguiente pregunta: ¿Acaso dicha población cercana a una zona minera no es peruana? ¿Acaso dichos peruanos, tienen menos derechos que los que viven en zonas de mayor comercio y actividad económica? ¿Es justo que la población con menos acceso a mejores oportunidades de vida y crecimiento personal y profesional continúe en esas condiciones de vida?

He ahí donde debe surgir el interés de responsabilidad social que deben tener las empresas del país, pues no es correcto ni posible, que una empresa minera se instale, desarrolle sus procesos y no tenga una actividad propositiva y de aporte para la población que habite a los alrededores suyos. Para la minera Pierina y sus autoridades ha sido prácticamente imposible tan si quiera tener la idea de no aportar a la población cercana, es por esto que han provisto la implementación de diferentes proyectos de responsabilidad social y de apoyo a la comunidad, la cual percibe un respaldo del sector privado, aun cuando la función principal debiera ser planificada y llevada a cabo por parte del Estado, aun sabiendo que recibe dinero producto de la cuota que la empresa le adjunta al gobierno por las acciones que desarrolla y por el material que extrae y exporta.

Es tal el aporte de la empresa que aun cuando no ha habido programas estatales que lleguen hasta la zona, esta ha proyectado un plan de alfabetización para todas las personas hasta 100 kilómetros de radio de distancia, totalmente gratuito; así como también ha desarrollado un plan de vacunación contra diversas enfermedades que se presentan constantemente en la zona y han cobrado muchas vidas, generando y aplicando planes de prevención que aborden dicha problemática y la subsanen; por otra parte, también han puesto en marcha un programa de becas para que los estudiantes más sobresalientes puedan tener acceso a estudios de mejores condiciones en la capital, incluso hasta en el extranjero, brindando a dichos estudiantes que continúen con los mejores niveles de rendimiento académico oportunidades de seguir estudiando a nivel universitario en las mejores universidades o institutos del mundo, según el plan de vida que el estudiante escoja para sí.

En muchas otras áreas, la empresa minera Pierina ha ayudado a la población que vive a sus alrededores construyendo colegios, trayendo docentes de las ciudades más cercanas y también grandes profesionales destacados en pedagogía y en didáctica, tanto del nivel educativo inicial, primario y secundario en las distintas especialidades, brindando a la comuna más cercana una educación de mejor calidad, tratando se sustituir en gran medida lo que el estado no puede llegar a cubrir, en tanto la población espera que el gobierno central, regional o local aborde sus problemáticas desde una visión que les visibilice y de importancia, dejando atrás todo razonamiento absurdo que deje de considerarles y pasen a ser los principales beneficiados por la riqueza del territorio al cual tienen cerca (Dionicio et al., 2008)

\subsection{Beneficios que aporta la empresa minera a las comunidades (ver tabla 3 )}

Ahora bien, se tienen opiniones diversas de acuerdo con los habitantes de la comunidad a los que se le encueste, siendo el caso de que, para la comunidad Cuncashca un $75 \%$ señala que fueron beneficiarios directos del Programa PIRSA que emprende la empresa Barrick, de igual forma un $72.5 \%$ de los encuestados de la comunidad Mareniyoc señalan lo mimo a igual que $65.5 \%$ de Atupa y el $63.5 \%$ respectivamente Mataquita.

\subsection{Desarrollo sostenible de la empresa Barrick hacia las comunidades}

Cabe señalar que como se indicó con anterioridad la muestra seleccionada fue un total de 75 hogares, los cuales están dispersos de la siguiente forma: Mataquita (40), Mareniyoc (16), Atupa (11), y Cuncashca (8). A los que se le aplicó el instrumento y se obtuvo como resultado que en promedio el $70 \%$ de la población vulnerable señala que mediante el programa PIRSA de la empresa si se benefició. No obstante, se presentan datos relevantes como en relación con la alfabetización, que "si se proyecta el PBI y la tasa de alfabetismo de la población, según las tendencias de progreso de los años 1950-1978, se aprecia cómo el PBI del 2009 debió haberse alcanzado hace 4 o 5 años, pero la tasa de alfabetismo actual debió haberse alcanzado hace 15 años". (Luis Alberto Orihuela Salazar, 2019), cosa que no se relaciona con la realidad, como se ha indicado, datos arriba. Otro aspecto que se debe considerar es la situación sociopolítica general del Perú que incide en los aspectos locales de las comunidades, y también en el desempeño de los programas sociales.

Sin embargo, la realidad local con el impacto de la mina Pierina, la cual cabe mencionar inicia sus actividades en 1998, ha sido cambiada en base a su presencia, pues incentiva, acelera de alguna forma los elementos económicos y sociales de la región, por lo que se tiene que citando a Orihuela:

Tabla 3. Beneficios que aporta la empresa minera a las comunidades

\begin{tabular}{ccccccccc}
\hline $\begin{array}{c}\text { Comunidad } \\
\text { Respuesta }\end{array}$ & \multicolumn{2}{c}{ Mataquita } & \multicolumn{2}{c}{ Mareniyoc } & \multicolumn{2}{c}{ Atupa } & \multicolumn{2}{c}{ Cuncashca } \\
FI & Porcentaje & Fx & Porcentaje & Fx & Porcentaje & Fx & Porcentaje \\
NO & 127 & $63.5 \%$ & 58 & $72.5 \%$ & 36 & $65.5 \%$ & 30 & $75.0 \%$ \\
No sabe / No responde & 68 & $34.0 \%$ & 20 & $25.0 \%$ & 17 & $30.9 \%$ & 9 & $22.5 \%$ \\
Total & 5 & $2.5 \%$ & 2 & $2.5 \%$ & 2 & $3.6 \%$ & 1 & $2.5 \%$ \\
\hline
\end{tabular}

Fuente: Elaboración propia 
En cuanto a todo lo referente a los aportes significativos de la mina para con el aspecto socioeconómico a destacar se puede referenciar las siguientes acciones y logros correspondientes:

- Se ha reducido los índices de pobreza con los que la población de Jangas contaba, tal reducción ha permitido pasar de un $80 \%$ a cerca de un $31 \%$, lo cual es altamente significativo pues le confiere al poblador tener una mayor esperanza de vida y calidad de la misma.

- Cuando la mina Pierina inició sus acciones los indicadores de pobreza extrema llegaban a marcar un $17,2 \%$, los cuales con el accionar positivo y en cumplimiento estricto de la Ley pudieron reducirse significativamente, tanto así que, al realizar el análisis en el año 2007, llegando a reducir 7 puntos porcentuales, lo cual evidencia el compromiso social de la empresa minera para las familias y su territorio.

- Disminuyo la tasa de analfabetismo en Jangas: del $44,8 \%$ al $23,6 \%$.

- En el área de influencia de Pierina se construyeron aproximadamente 8.400 viviendas, es decir, 600 viviendas por año aproximadamente.

- Al 2007, un 94\% de las viviendas de Jangas disponía de agua potable todos los días, mientras que en el conjunto de Ancash ese indicador llegaba al $90,0 \%$.

- Los ocupados aumentaron en más de 10 mil personas.

- La población sin documento nacional de identidad o sin partida de nacimiento se redujo a niveles mínimos en el área de influencia (Luis Alberto Orihuela Salazar, 2019)

Es importante señalar, que este estudio se enfoca en lo consolidado por la empresa, en particular, quien tiene como política de ayuda, la de apoyar actividades económicas, que desde su concepción sean productivas en el tiempo o de forma permanente para estas poblaciones, se tiene que, entre las observaciones realizadas por el estudio en que se basa este artículo, las obras más destacadas son: un programa de dotación de útiles escolares con bolsos, dotación de una sala de cómputo para la secundaria local, y un taller de carpintería. De igual forma ha dotado de servicios de energía eléctrica, agua potable, reservorios de agua y silos que impactan de manera significativa en la calidad de vida de la población. Otro aspecto por considerar es que, la empresa ofrece trabajos menores de tipo rotativo a las comunidades, los cuales pueden tener un periodo de duración en promedio de tres meses, representando una remuneración mensual de aproximadamente 515 nuevos soles. Todo ello se puede ver traducido en la siguiente tabla 4.

De todo ello se puede inferir que las acciones llevadas a cabo por la empresa minera la cual es objeto de estudio han sido positivas en tanto han redundado en bienestar y mejores condiciones de vida para las poblaciones vulnerables que viven en las zonas cercanas al asentamiento minero.

\section{CONCLUSIONES}

El accionar de la Empresa Barrick, representa para los habitantes de las comunidades aledañas a la misma donde han puesto en marcha el programa emblema, un beneficio significativo, ya que sus programas de desarrollo social y ambiental han traído cambios significativos y que se traducen en un alto nivel de satisfacción para los encuestados.

Algo que se debe tomar en consideración es el alcance de los programas de la empresa minera pues los mismos han conllevado a la difusión de conocimientos relativos al fomento de "una cultura ambiental" no solo sus empleados, sino a las comunidades que influencia, teniendo entre los principales aspectos positivos, el crecimiento demográfico, proyectos de inversión sostenibles, y un incentivo que va más allá del aspecto netamente económico y permitir el desarrollo sostenible de la comunidades que se desarrollan en la periferia de la mina.

En base a lo anteriormente expresado, se puede decir que, la empresa Barrick Misquichilca S.A., debe dar continuidad a su Programa PIRSA, ya que ha sido abierta y significativamente beneficioso para las poblaciones del Distrito de Jangas, Huaraz, pues el mismo representa un elemento de beneficio social para los habitantes de la zona.

\section{AGRADECIMIENTOS}

A la Universidad Nacional Mayor de San Marcos, a la Unidad de postgrado de la Facultad de Ingeniería Geológica, Minera, Metalúrgica y Geográfica, a mi asesor,

Tabla 4. Compromiso y bienestar de la empresa Barrick con las comunidades

\begin{tabular}{|c|c|c|c|c|c|c|c|c|}
\hline \multirow{2}{*}{$\begin{array}{l}\text { Comunidad } \\
\text { Respuesta }\end{array}$} & \multicolumn{2}{|c|}{ Mataquita } & \multicolumn{2}{|c|}{ Mareniyoc } & \multicolumn{2}{|c|}{ Atupa } & \multicolumn{2}{|c|}{ Cuncashca } \\
\hline & $\mathrm{Fx}$ & Porcentaje & $F x$ & Porcentaje & $F x$ & Porcentaje & Fx & Porcentaje \\
\hline SI & 136 & 68.0 & 58 & 72.5 & 38 & 69.1 & 30 & 75.0 \\
\hline NO & 61 & 30.5 & 20 & 25.0 & 16 & 29.1 & 8 & 20.0 \\
\hline No sabe / No responde & 3 & 1.5 & 2 & 2.5 & 1 & 1.8 & 2 & 5.0 \\
\hline Total & 200 & $100 \%$ & 80 & $100 \%$ & 55 & $100 \%$ & 40 & $100 \%$ \\
\hline
\end{tabular}

Fuente: Elaboración propia 
el Dr. Carlos Cabrera Carranza, por su guía y conocimiento en el desarrollo de esta investigación. Al Dr. Vidal Sixto Aramburú Rojas por apoyarme con su experiencia profesional.

\section{REFERENCIAS}

Dionicio, E., Cabrera, C., Figueroa, A., \& Caballero, M. (2008). Sostenibilidad ambiental y responsabilidad social en la actividad aurífera: el caso de Pierina (Ancash). Revista Del Instituto de Investigación de La Facultad de Ingeniería Geológica, Minera, Metalurgica y Geográfica, 11(21), 2531. https://revistasinvestigacion.unmsm.edu.pe/index.php/ iigeo/article/view/481

Espinoza Guadalupe, L. (2004). La gestión de responsabilidad social empresarial de las empresas mineras en el Perú y su incidencia en el desarrollo sostenible de las comunidades de su entorno - período 2004 - 2014. In REPOSITORIO ACADÉMICO USMP. https://repositorio.usmp.edu.pe/ handle/20.500.12727/1857

Hernández-Sampieri, R. (2014). Metodología de la Investigación (Mc Graw Hill. México, Ed.). http://observatorio. epacartagena.gov.co/wp-content/uploads/2017/08/ metodologia-de-la-investigacion-sexta-edicion.compressed. pdf

INEI. (2019). Panorama de la Economía Peruana: 1950-2018. Instituto Nacional de Estadística e Informática, 175. https:// www.inei.gob.pe/media/MenuRecursivo/publicaciones digitales/Est/Lib1654/libro.pdf
Juan Antonio Gil Pascual. (2016). Técnicas e instrumentos para la recogida de información. https://books.google.com.pe/ books?hl=es\&lr=\&id=ANrkDAAAQBAJ\&oi=fnd\&pg=PP $1 \& \mathrm{dq}=$ Gil + Pascual, + J. + A. $+(2016) .+\mathrm{T} \% \mathrm{C} 3 \%$ A9cnicas $+\mathrm{e}+$ instrumentos + para + la + recogida + de + informaci $\% \mathrm{C} 3$ $\%$ B3n.+Universidad\%09Nacional\%09de\%09Educaci $\% \mathrm{C} 3 \% \mathrm{~B} 3 \mathrm{n} \% 09 \mathrm{a} \% 09 \mathrm{Distancia} .+\mathrm{UNED} \&$ ots $=$ rb6np Mm7GJ\&sig=tXx0JAVOdCaI6E4aEzfJhbHa8bk\#v=one page $\& q \& \mathrm{f}=$ false

Luis Alberto Orihuela Salazar. (2019). La responsabilidad social $y$ ambiental de la industria minera en el Perú. Caso: Minera Barrick Misquichilca - Pierina. https://cybertesis.unmsm. edu.pe/bitstream/handle/20.500.12672/11331/Orihuela_ sl.pdf? sequence $=1 \&$ isAllowed $=\mathrm{y}$

Sergio Carrasco Díaz. (2005). Metodología de la investigación cientifica: Pautas metodológicas para diseñar y elaborar el proyecto de investigación. https://sisbiblio.unah.edu.pe/ opac_css/index.php?lvl=notice_display\&id $=490$

Spiegel, M., \& Stephens, L. (2009). Estadística 3era Edición. The McGraw-Hill. https://www.academia.edu/36241872/ Estadística_Serie_Schaum_4ta_edición_Murray_R_ Spiegel_pdf_1_1_ 